\title{
Neutrino evolution in external environments: New effects
}

\author{
Alexander Studenikin* \\ Lomonosov Moscow State University, 119992 Moscow, Russia \\ Joint Institute for Nuclear Research, 141980 Dubna, Moscow Region, Russia \\ E-mail: studenikdsrd.sinp.msu.ru
}

\begin{abstract}
During a period of about two decades we have realized a programme of systematic investigations of different aspects of neutrino propagation in extreme external environments and have predicted and studied several new phenomena that are engendered by the presence of external magnetic fields and dense matter. The starting point that underlies the research is the fact that the electromagnetic properties of neutrinos open a window to new physics [1-3]. In these brief notes, we recall several new phenomena that have been proposed and investigated earlier. In particular, we discuss: 1) the spin light of neutrino in matter, 2) the neutrino energy quantization in rotating matter, and 3) neutrino start turning mechanism. Then we dwell on results of recent studies: 4) the effects of interplay of neutrino flavour and spin oscillations in a magnetic field, 5) the quantum theory of neutrino spin and spin-flavour oscillations engendered by the transversal mater currents, 6) the amplitude modulation of the flavour neutrino oscillation probability by the transversal matter current. As for references to the literature, only those papers are included in which a particular effect was proposed and considered for the first time and in a number of cases references are also be given to the most recent articles, which contain detailed references to the available literature on the issue.
\end{abstract}

European Physical Society Conference on High Energy Physics - EPS-HEP2019 -

10-17 July, 2019

Ghent, Belgium

\footnotetext{
* Speaker.
} 


\section{Neutrino spin light and energy quatization in magnetized matter}

Spin light of neutrino. The spin light of neutrino $(S L v)$ is a new mechanism of electromagnetic radiation emitted by a massive neutrino (with a nonzero magnetic moment) moving in external media that was proposed in [4] on the basis of the quaziclassical theory of the neutrino spin evolution in matter. The quantum theory of the spin light of neutrino in matter was developed in [5] and it was shown that $S L v$ originates from the neutrino-photon magnetic moment coupling and from the energy splitting, induced in an external environment, of two different neutrino helicity states characterized by $s= \pm 1$ (see [5-9] and references therein). The total rate and power of the radiation in homogeneous neutron matter (far from the threshold), $\Gamma=2 \mu^{2} G_{F}^{2} n^{2} p, \quad I={ }^{2} / 3 \mu^{2} G_{F}^{2} n^{2} p^{2}$, exhibit strong dependance on the neutrino energy and matter density $n$ ( $p$ and $\mu$ are the neutrino momentum and magnetic moment). The $S L v$ radiation has also a non-trivial polarization properties $[5,6]$. In the nuclear matter, the $S L v$ of an ultrarelativistic neutrino is completely circular polarized.

Although this effect is very weak due to smallness of the neutrino magnetic moment, it can be of interest for astrophysical environments involving compact relativistic objects because its efficiency is higher, the higher the neutrino energy and background matter density. In our recent studies $[8,9]$ we summarize conditions for best $S L v$ efficiency in astrophysical settings and conclude that the most suitable astrophysical site for manifestation of this phenomenon is represented by short Gamma-Ray Bursts (see, for instance, [10]) where generation of ultra-high energy neutrinos is anticipated and the matter density can be of the order of the nuclear density.

Neutrino energy quantization in rotating matter. Another interesting phenomenon is the neutrino energy quantization [11] in the case the particle propagation in a rotating medium. Using the method of exact solutions for the neutrino wave functions in the external matter [12] it was shown that the neutrino $v_{e}$ energy spectrum in a rotating and magnetized matter composed of neutrons is given by [11,13-15]: $p_{0}=\sqrt{p_{3}^{2}+2 N\left(q_{v} B+2 G n_{n} \omega\right)}-G n_{n}, \quad G=\frac{G_{F}}{\sqrt{2}}$, where $N$ is an integer number, $n_{n}$ is the neutron invariant number density (it is supposed that the direction of the neutrino propagation coincides with direction of the magnetic field $\boldsymbol{B}$ and the matter rotation angular velocity $\boldsymbol{\omega}$ ). The energy spectrum is quantized due to both weak and electromagnetic interactions (due to possible nonzero neutrino millicharge $q_{v}$ ) and represents the modified Landau levels of the millicharged neutrino in the rotating magnetized matter. In the quasiclassical treatment neutrino are moving on circular orbits with radius given by $R=\Omega^{-1}$, where the effective rotation frequency $\Omega=\omega_{m}+\omega_{c}$ is determined [14] by the cyclotron frequency $\omega_{c}=\frac{q_{v} B}{p_{0}+G n_{n}}$ and the matter induced frequency $\omega_{m}=\frac{2 G n_{n}}{p_{0}+G n_{n}} \omega$.

It is possible to explain [12] the neutrino quasiclassical circular orbits as a result of action of the attractive central force, $\mathbf{F}_{m}^{(v)}=q_{m}^{(v)} \boldsymbol{\beta} \times \mathbf{B}_{m}, \mathbf{B}_{m}=\boldsymbol{\nabla} \times \mathbf{A}_{m}, \mathbf{A}_{m}=n \mathbf{v}$, where the effective neutrino "charge" in matter (composed of neutrons in the discussed case) is $q_{m}^{(v)}=-G$, whereas $\mathbf{B}_{m}$ and $\mathbf{A}_{m}$ play the roles of effective "magnetic" field and the correspondent "vector potential". Like the magnetic part of the Lorentz force, $\mathbf{F}_{m}^{(v)}$ is orthogonal to the neutrino speed $\boldsymbol{\beta}$. For the most general case (when the matter density $n$ is not constant) the "matter induced Lorentz force" is given by [14] $\mathbf{F}_{m}^{(v)}=q_{m}^{(v)} \mathbf{E}_{m}+q_{m}^{(v)} \boldsymbol{\beta} \times \mathbf{B}_{m}$, where the effective "electric" and "magnetic" fields are respectively, $\mathbf{E}_{m}=-\boldsymbol{\nabla} n-\mathbf{v} \frac{\partial n}{\partial t}-n \frac{\partial \mathbf{v}}{\partial t}, \quad \mathbf{B}_{m}=n \boldsymbol{\nabla} \times \mathbf{v}-\mathbf{v} \times \boldsymbol{\nabla} n$, here $\mathbf{v}$ is the speed of matter. The force acting on a neutrino, produced by the first term of the effective "electric" field in the neutron matter, was considered in [16], similar quasiclassical treatment of a neutrino motion in the electron 
plasma was considered in [17].

Neutrino start turning mechanism. The effect of the millicharged neutrino energy quantization and the corresponding neutrino motion on curved orbits in a magnetized matter applied for neutrinos propagating inside rotating neutron stars provides the best astrophysical bound for the neutrino millicharge [14]. In the the magnetized matter the effective Lorentz force discussed in the previous section disturbs the neutrino trajectories. Obviously, the feedback of the escaping millicharged neutrinos that are moving on curved orbits inside a magnetized rotating star should effect initial star rotation. We have termed this phenomenon the "Neutrino Star Turning" ( $v S T)$ mechanism. In order to avoid the contradiction of $v S T$ mechanism impact with observational data on pulsars a stringent limit on the neutrino millichager was obtained [14]: $q_{v}<1.3 \times 10^{-19} e_{0}$, that is indeed the best astrophysical upper bound on $q_{v}$.

\section{New effect of neutrino oscillations}

Interplay of neutrino flavour, spin and spin-flavour oscillations in a constant magnetic field. A new approach to description of neutrino spin and spin-flavor oscillations in the presence of an arbitrary constant magnetic field has been developed $[18,19]$ recently. Within the new approach exact quantum stationary states are used for classification of neutrino spin states, rather than the neutrino helicity states that are used for this purpose within the customary approach in many published papers. Recall that the helicity states are not stationary in the presence of a magnetic field. It has been shown [20] in particular, that in the presence of the transversal magnetic field $B_{\perp}$ for a given choice of parameters (the energy and magnetic moments of neutrinos and strength of the magnetic field) the amplitude of the flavour oscillations $v_{e}^{L} \Leftrightarrow v_{\mu}^{L}$ at the vacuum frequency $\omega_{v a c}=\frac{\Delta m^{2}}{4 p}$ is modulated by the magnetic field frequency $\omega_{B}=\mu B_{\perp}$ :

$$
P_{v_{e}^{L} \rightarrow v_{\mu}^{L}}^{\left(B_{\perp}\right)}(t)=\left(1-\sin ^{2}\left(\mu B_{\perp} t\right)\right) \sin ^{2} 2 \theta \sin ^{2} \frac{\Delta m^{2}}{4 p} t=\left(1-P_{v_{e}^{L} \rightarrow v_{e}^{R}}^{\text {cust }}\right) P_{v_{e}^{L} \rightarrow v_{\mu}^{L}}^{\text {cust }}
$$

here $\mu$ is the effective magnetic moment of the electron neutrino and it is supposed that the following relations between diagonal and transition magnetic moments in the neutrino mass basis are valid: $\mu_{1}=\mu_{2}, \quad \mu_{i j}=0, i \neq j$. The customary expression $P_{v_{e}^{L} \rightarrow v_{\mu}^{L}}^{\text {cust }}(t)=\sin ^{2} 2 \theta \sin ^{2} \frac{\Delta m^{2}}{4 p} t$ for the neutrino flavour oscillation probability in vacuum in the presence of the transversal field $B_{\perp}$ is modified by the factor $1-P_{v_{e}^{L} \rightarrow v_{e}^{R}}^{\text {cust }}$. Since the transition magnetic moment in the flavour basis is absent in the case $\mu_{1}=\mu_{2}$, the process $v_{e}^{L} \rightarrow v_{e}^{R}$ is the only way for spin flip, and then $1-P_{v_{e}^{L} \rightarrow v_{e}^{R}}^{\text {cust }}$ should be interpreted as the probability of not changing the neutrino spin polarization. And consequently, this multiplier subtracts the contribution of neutrinos $v_{e}^{R}$ with the opposite polarization providing the survival of the only contribution from the direct neutrino flavour oscillations $v_{e}^{L} \Leftrightarrow v_{\mu}^{L}$. Similar results on the important influence of the transversal magnetic field on amplitudes of various types of neutrino oscillations were obtained earlier [21] on the basis of the exact solution of the effective equation for neutrino evolution in the presence of a magnetic field, which accounts for four neutrino species corresponding to two different flavor states with positive and negative helicities.

Consider the probability of the neutrino spin-flavour oscillations $v_{e}^{L} \leftrightarrow v_{\mu}^{R}$. In the case $\mu_{1}=$ $\mu_{2}=\mu$ we have [20]:

$$
P_{v_{e}^{L} \rightarrow v_{\mu}^{R}}(t)=\sin ^{2}\left(\mu B_{\perp} t\right) \sin ^{2} 2 \theta \sin ^{2} \frac{\Delta m^{2}}{4 p} t .
$$


The obtained expression (2.2) for the probability can be expressed as a product of two probabilities derived within the customary two-neutrino-states approach

$$
P_{v_{e}^{L} \rightarrow v_{\mu}^{R}}(t)=P_{v_{e}^{L} \rightarrow v_{\mu}^{L}}^{\text {cust }}(t) P_{v_{e}^{L} \rightarrow v_{e}^{R}}^{\text {cust }}(t)
$$

where $P_{v_{e}^{L} \rightarrow v_{\mu}^{L}}^{c u s t}(t)=\sin ^{2} 2 \theta \sin ^{2} \frac{\Delta m^{2}}{4 p} t$ and the usual expression for the neutrino spin oscillation probability

$$
P_{v_{e}^{L} \rightarrow v_{e}^{R}}^{\text {cust }}(t)=\sin ^{2}\left(\mu B_{\perp} t\right)
$$

are just the probabilities obtained in the customary approach. A similar neutrino spin-flavour oscillations (for the Majorana case) as a two-step neutrino conversion processes were considered in [22] (however, the effect was calculated within perturbation theory since the probability of neutrino spin-flavour oscillations was supposed to be small).

Finally, in the case of the spin oscillations $v_{e}^{L} \rightarrow v_{e}^{R}$ in the transversal magnetic field $B_{\perp}$ within the the developed approach the effect of neutrino mixing is accounted for that leads to the modification of the customary expression $P_{v_{e}^{L} \rightarrow v_{e}^{R}}^{\text {cust }}(t)$ for the probability by the factor $1-P_{v_{e}^{L} \rightarrow v_{\mu}^{L}}^{\text {cust }}$ :

$$
P_{v_{e}^{L} \rightarrow v_{e}^{R}}=\left[1-\sin ^{2} 2 \theta \sin ^{2}\left(\frac{\Delta m^{2}}{4 p} t\right)\right] \sin ^{2}\left(\mu B_{\perp} t\right) .
$$

The interplay between different oscillations, that follows from the obtained expressions (‥]), (2.2) and ([2.5) for the oscillation probabilities, gives rise to interesting phenomena [20]: 1) the amplitude modulation of the probability of flavour oscillations $v_{e}^{L} \rightarrow v_{\mu}^{L}$ in the transversal magnetic field with the magnetic frequency $\omega_{B}=\mu B_{\perp}$ (in the case $\mu_{1}=\mu_{2}$ ) and more complicated dependence on the harmonic functions with $\omega_{B}$ for $\mu_{1} \neq \mu_{2} ; 2$ ) the dependence of the spin oscillation probability $P_{v_{e}^{L} \rightarrow v_{e}^{R}}$ on the mass square difference $\Delta m^{2}$; 3) the appearance of the spin-flavour oscillations in the case $\mu_{1}=\mu_{2}$ and $\mu_{12}=0$, the transition goes through the two-step processes $v_{e}^{L} \rightarrow v_{\mu}^{L} \rightarrow v_{\mu}^{R}$ and $v_{e}^{L} \rightarrow v_{e}^{R} \rightarrow v_{\mu}^{R}$.

As a result, we predict modifications of the neutrino oscillation patterns that might provide new important phenomenological consequences in case of neutrinos propagation in extreme astrophysical environments where magnetic fields are present.

Neutrino spin and spin-flavour oscillations in transversal matter current. Another new effect in neutrino oscillations was predicted in [23]: it was shown the the neutrino spin precession and oscillations can be engendered by neutrino interactions with transversally moving (or polarized) matter(even in the absence of a magnetic field). The existence of this effect was also confirmed in [24-28].

Initially the effect was predicted [23] within the semiclassical theory of the neutrino spin evolution in the transversal matter currents has been recently appended by the direct quantum treatment of the phenomenon $[29,30]$. Consider two flavour neutrinos with two possible helicities $v_{f}=\left(v_{e}^{+}, v_{e}^{-}, v_{\mu}^{+}, v_{\mu}^{-}\right)^{T}$ in moving matter composed of neutrons. Each of the flavour neutrinos is a superposition of the neutrino mass states, $v_{e}^{ \pm}=v_{1}^{ \pm} \cos \theta+v_{2}^{ \pm} \sin \theta, v_{\mu}^{ \pm}=-v_{1}^{ \pm} \sin \theta+v_{2}^{ \pm} \cos \theta$. The corresponding neutrino evolution equation is

$$
i \frac{d}{d t} v_{f}=H_{v}^{f} v_{f}
$$


where the evolution Hamiltonian reads

$$
H_{v}^{f}=n \tilde{G}\left(\begin{array}{cccc}
0 & \left(\frac{\eta}{\gamma}\right)_{e e} v_{\perp} & 0 & \left(\frac{\eta}{\gamma}\right)_{e \mu} v_{\perp} \\
\left(\frac{\eta}{\gamma}\right)_{e e} v_{\perp} & 2\left(1-v_{\|}\right) & \left(\frac{\eta}{\gamma}\right)_{e \mu} v_{\perp} & 0 \\
0 & \left(\frac{\eta}{\gamma}\right)_{e \mu} v_{\perp} & 0 & \left(\frac{\eta}{\gamma}\right)_{\mu \mu} v_{\perp} \\
\left(\frac{\eta}{\gamma}\right)_{e \mu} v_{\perp} & 0 & \left(\frac{\eta}{\gamma}\right)_{\mu \mu} v_{\perp} & 2\left(1-v_{\|}\right)
\end{array}\right)
$$

and

$$
\left(\frac{\eta}{\gamma}\right)_{e e}=\frac{\cos ^{2} \theta}{\gamma_{11}}+\frac{\sin ^{2} \theta}{\gamma_{22}},\left(\frac{\eta}{\gamma}\right)_{\mu \mu}=\frac{\sin ^{2} \theta}{\gamma_{11}}+\frac{\cos ^{2} \theta}{\gamma_{22}},\left(\frac{\eta}{\gamma}\right)_{e \mu}=\frac{\sin 2 \theta}{\tilde{\gamma}_{21}},
$$

where $\widetilde{\gamma}_{21}^{-1}=\frac{1}{2}\left(\gamma_{2}^{-1}-\gamma_{1}^{-1}\right), \gamma_{\alpha}^{-1}=\frac{m_{\alpha}}{p_{0}^{v}}, p_{0}^{v}$ is neutrino energy and $\left.\alpha=1,2\right)$.

Consider the initial neutrino state $v_{e}^{L}$ moving in the background with the magnetic field $\boldsymbol{B}=$ $\boldsymbol{B}_{\|}+\boldsymbol{B}_{\perp}$ and nonzero matter current $\boldsymbol{j}=\boldsymbol{j}_{\|}+\boldsymbol{j}_{\perp}$. One of the possible modes of neutrino transitions with the change of helicity is $v_{e}^{L} \Leftarrow\left(j_{\perp}, B_{\perp}\right) \Rightarrow v_{e}^{R}$. The corresponding oscillations are governed by the evolution equation [30]

$$
i \frac{d}{d t}\left(\begin{array}{c}
v_{e}^{L} \\
v_{e}^{R}
\end{array}\right)=\left(\begin{array}{cc}
\left(\frac{\mu}{\gamma}\right)_{e e} B_{\|}+\widetilde{G} n(1-\boldsymbol{v} \boldsymbol{\beta}) & \mu_{e e} B_{\perp}+\left(\frac{\eta}{\gamma}\right)_{e e} \widetilde{G} n v_{\perp} \\
\mu_{e e} B_{\perp}+\left(\frac{\eta}{\gamma}\right)_{e e} \widetilde{G} n v_{\perp} & -\left(\frac{\mu}{\gamma}\right)_{e e} B_{\|}-\widetilde{G} n(1-\boldsymbol{v} \boldsymbol{\beta}
\end{array}\right)\left(\begin{array}{c}
v_{e}^{L} \\
v_{e}^{R}
\end{array}\right) .
$$

Here we constraint our consideration to the binary neutrino transitions and corresponding oscillations between pairs of the neutrino states. The oscillation $v_{e}^{L} \Leftarrow\left(j_{\perp}, B_{\perp}\right) \Rightarrow v_{e}^{R}$ probability is given by

$$
P_{v_{e}^{L} \rightarrow v_{e}^{R}}^{\left(j_{\perp}, B_{\perp}\right)}(x)=\frac{E_{\mathrm{eff}}^{2}}{E_{\mathrm{eff}}^{2}+\Delta_{\mathrm{eff}}^{2}} \sin ^{2} \frac{\pi x}{L_{\mathrm{eff}}}, \quad L_{\mathrm{eff}}=\frac{\pi}{\sqrt{E_{\mathrm{eff}}^{2}+\Delta_{\mathrm{eff}}^{2}}},
$$

where

$$
E_{e f f}=\left|\mu_{e e} \boldsymbol{B}_{\perp}+\left(\frac{\eta}{\gamma}\right)_{e e} \widetilde{G} n \boldsymbol{v}_{\perp}\right|, \quad \Delta_{e f f}=\left|\left(\frac{\mu}{\gamma}\right)_{e e} \boldsymbol{B}_{\|}+\widetilde{G} n(1-\boldsymbol{v} \boldsymbol{\beta}) \boldsymbol{\beta}\right| .
$$

From (2.10) and (2.1) it is clearly seen that even in the absence of the transversal magnetic field the neutrino spin oscillations $v_{e}^{L} \Leftarrow\left(j_{\perp}\right) \Rightarrow v_{e}^{R}$ can be generated by the neutrino interaction with the transversal matter current $\boldsymbol{j}_{\perp}=n \boldsymbol{v}_{\perp}$.

In a quite analogous way the transversal matter current can engender the neutrino spin-flavour conversion and the corresponding oscillations $v_{e}^{L} \Leftarrow\left(j_{\perp}\right) \Rightarrow v_{\mu}^{R}$ between two different flavour states with opposite spin orientations. Note that for apperance of this effect there is no need for a magnetic field (obviously, the effect is neutrino magnetic moments independent). For the neutrino evolution $v_{e}^{L} \Leftarrow\left(j_{\perp}\right) \Rightarrow v_{\mu}^{R}$ in the case $B=0$ from (‥7) we get

$$
i \frac{d}{d t}\left(\begin{array}{c}
v_{e}^{L} \\
v_{\mu}^{R}
\end{array}\right)=\left(\begin{array}{cc}
-\Delta M+\widetilde{G} n(1-\boldsymbol{v} \boldsymbol{\beta}) & \left(\frac{\eta}{\gamma}\right)_{e \mu} \widetilde{G} n v_{\perp} \\
\left(\frac{\eta}{\gamma}\right)_{e \mu} \widetilde{G} n v_{\perp} & \Delta M-\widetilde{G} n(1-\boldsymbol{v} \boldsymbol{\beta})
\end{array}\right)\left(\begin{array}{c}
v_{e}^{L} \\
v_{\mu}^{R}
\end{array}\right),
$$

where $\Delta M=\frac{\Delta m^{2} \cos 2 \theta}{4 p_{0}^{v}}$. The probability $P_{v_{e}^{L} \rightarrow v_{\mu}^{R}}^{\left(j_{\perp}\right)}$ of the neutrino spin-flavour oscillations $v_{e}^{L} \Leftarrow$ $\left(j_{\perp}\right) \Rightarrow v_{\mu}^{R}$ is given by the same equation (2. $(2)$ ), but now

$$
E_{e f f}=\left|\left(\frac{\eta}{\gamma}\right)_{e \mu} \widetilde{G} n v_{\perp}\right|, \Delta_{e f f}=|\Delta M-\widetilde{G} n(1-\boldsymbol{v} \boldsymbol{\beta})| .
$$


From ([.]3]) it follows that the neutrino spin-flavour oscillations $v_{e}^{L} \Leftarrow\left(j_{\perp}\right) \Rightarrow v_{\mu}^{R}$ can be generated by the neutrino interaction with the transversal matter current $\boldsymbol{j}_{\perp}=n \boldsymbol{v}_{\perp}$.

Amplitude modulation of flavour neutrino oscillation probability by transversal matter current. From the previous discussions (see [20] for details) it follows that in the presence of a magnetic field it is not possible to consider the neutrino flavour and spin oscillations as separate phenomena. On the contrary, there is an inherent communication between two. In particular, the amplitude of the neutrino flavour oscillations is modulated by the magnetic frequency $\omega_{B}=\mu B_{\perp}$. The main result of the above discussion (see also [23,30]) is the conclusion on the equal role that the transversal magnetic field $\boldsymbol{B}_{\perp}$ and the transversal matter current $\boldsymbol{j}_{\perp}$ plays in generation of the neutrino spin and spin-flavour oscillations. From these observations we predict a new phenomenon of the modification of the flavour neutrino oscillations probability in moving matter under the condition of non-vanishing matter transversal current $j_{\perp}=n v_{\perp}$.

The flavour neutrino oscillation probability accounting for this effect can be expressed as follows:

$$
P_{v_{e}^{L} \rightarrow v_{\mu}^{L}}^{\left(j_{\|}+j_{\perp}\right)}(t)=\left(1-P_{v_{e}^{L} \rightarrow v_{e}^{R}}^{\left(j_{\perp}\right)}-P_{v_{e}^{L} \rightarrow v_{\mu}^{R}}^{\left(j_{\perp}\right)}\right) P_{v_{e}^{L} \rightarrow v_{\mu}^{L}}^{\left(j_{\|}\right)},
$$

where $P_{v_{e}^{L} \rightarrow v_{\mu}^{L}}^{\left(j_{\|}\right)}(t)=\sin ^{2} 2 \theta_{e f f} \sin ^{2} \omega_{e f f} t$, is the flavour oscillation probability in moving matter [31], $\omega_{e f f}=\frac{\Delta m_{e f f}^{2}}{4 p_{0}^{v}}, \theta_{e f f}$ and $\Delta m_{e f f}^{2}$ are the corresponding quantities modified by the presence of moving matter (note that in the definition of $\theta_{e f f}$ and $\Delta m_{e f f}^{2}$ only the longitudinal component of matter motion matters). For the probability of the neutrino spin oscillations engendered by the transversal current $j_{\perp}$ from (2.9) we get

$$
P_{v_{e}^{L} \rightarrow v_{e}^{R}}^{j_{\perp}}(t)=\frac{\left(\frac{\eta}{\gamma}\right)_{e e}^{2} v_{\perp}^{2}}{\left(\frac{\eta}{\gamma}\right)_{e e}^{2} v_{\perp}^{2}+(1-v \boldsymbol{\beta})^{2}} \sin ^{2} \omega_{e e}^{j_{\perp}} t
$$

For the corresponding probability of the neutrino spin-flavour oscillations due to $\boldsymbol{j}_{\perp}$ from (2.13) we get

$$
P_{v_{e}^{L} \rightarrow v_{\mu}^{R}}^{j_{\perp}}(t)=\frac{\left(\frac{\eta}{\gamma}\right)_{e \mu}^{2} v_{\perp}^{2}}{\left(\frac{\eta}{\gamma}\right)_{e \mu}^{2} v_{\perp}^{2}+\left(\frac{\Delta M}{\widetilde{G} n}-(1-v \boldsymbol{\beta})\right)^{2}} \sin ^{2} \omega_{e \mu}^{j_{\perp} t} .
$$

The discussed new effect of the modification of the flavour oscillations $v_{e}^{L} \Leftarrow\left(j_{\|}, j_{\perp}\right) \Rightarrow v_{\mu}^{L}$ probability is the result of an interplay of oscillations on a customary flavour oscillation frequency in moving matter $\omega_{\text {eff }}$ and two additional oscillations with changing the neutrino polarization, the neutrino spin $v_{e}^{L} \Leftarrow\left(j_{\perp}\right) \Rightarrow v_{e}^{R}$ and spin-flavour $v_{e}^{L} \Leftarrow\left(j_{\perp}\right) \Rightarrow v_{\mu}^{R}$ oscilations, that are governed by two characteristic frequencies:

$$
\omega_{e e}^{j_{\perp}}=\widetilde{G} n \sqrt{\left(\frac{\eta}{\gamma}\right)_{e e}^{2} v_{\perp}^{2}+(1-\boldsymbol{v} \boldsymbol{\beta})^{2}}, \quad \omega_{e \mu}^{j_{\perp}}=\widetilde{G} n \sqrt{\left(\frac{\eta}{\gamma}\right)_{e \mu}^{2} v_{\perp}^{2}+\left(\frac{\Delta M}{\widetilde{G} n}-(1-\boldsymbol{v} \boldsymbol{\beta})\right)^{2}} .
$$

The discussed interplay of oscillations on different frequencies should be accounted for in analysis of propagation of neutrino fluxes in astrophysical environments. 


\section{References}

[1] C. Giunti and A. Studenikin, Rev. Mod. Phys. 87 (2015) 531.

[2] A. Studenikin, Nucl. Phys. Proc. Suppl. 188 (2009) 220.

[3] A. Studenikin, PoS EPS-HEP2017 (2017) 137

[4] A. Lobanov and A. Studenikin, Phys. Lett. B 564 (2003) 27.

[5] A. Studenikin and A. Ternov, Phys. Lett. B 608 (2005) 107 [hep-ph/0410297].

[6] A. Grigoriev, A. Studenikin and A. Ternov, Phys. Lett. B 622 (2005) 199.

[7] A. E. Lobanov, Phys. Lett. B 619 (2005) 136 [hep-ph/0506007].

[8] A. Grigoriev, A. Lokhov, A. Studenikin and A. Ternov, Phys. Lett. B 718 (2012) 512.

[9] A. Grigoriev, A. Lokhov, A. Studenikin and A. Ternov, JCAP 1711 (2017) 024.

[10] A. Perego at al, Mon. Not. R. Astron. Soc. 443 (2014) 3134.

[11] A. Grigoriev, A. Savochkin, A. Studenikin, Russ. Phys. J. 50 (2007) 845.

[12] A. Studenikin, J. Phys. A 41 (2008) 164047 [arXiv:0804.1417 [hep-ph]].

[13] I. Balantsev, Y. Popov and A.Studenikin, J. Phys. A 44 (2011) 255301.

[14] A. Studenikin and I. Tokarev, Nucl. Phys. B 884 (2014) 396.

[15] M. Dvornikov, JCAP 1505 (2015) 037.

[16] A. Loeb Phys.Rev.Lett. 64 (1990) 115.

[17] L. Oliveira e Silva, et al Phys. Lett. A 270 (2000) 265.

[18] A. Dmitriev, R. Fabbricatore and A. Studenikin, PoS CORFU 2014 (2015) 050.

[19] A. Studenikin, EPJ Web Conf. 125 (2016) 04018.

[20] A. Popov and A. Studenikin, Eur. Phys. J. C 79 (2019) 144.

[21] P. Kurashvili, K. Kouzakov, L. Chotorlishvili and A. Studenikin, Phys.Rev. D 96 (2017) 103017.

[22] E. Akhmedov and J. Pulido, Phys. Lett. B 553 (2003) 7.

[23] A. Studenikin, Phys. Atom. Nucl. 67 (2004) 993.

[24] V. Cirigliano, G. Fuller and A. Vlasenko, Phys. Lett. B 747 (2015) 27.

[25] C. Volpe, Int. J. Mod. Phys. E 24 (2015) 1541009.

[26] A.Kartavtsev, G.Raffelt and H.Vogel, Phys. Rev. D 91 (2015) 125020.

[27] A.Dobrynina, A. Kartavtsev and G.Raffelt Phys. Rev. D 93 (2016) 125030.

[28] J.-Y. Tian, A. Patwardhan and G.Fuller, Phys. Rev. D 95 (2017) 063004.

[29] A. Studenikin, J. Phys. Conf. Ser. 888 (2017) 012221.

[30] P. Pustoshny and A. Studenikin, Phys. Rev. D 98 (2018) 113009.

[31] A. Grigoriev, A. Lobanov and A. Studenikin, Phys. Lett. B 535 (2002) 187. 